\title{
Convergent evolution of sexual dimorphism in species of the family Dolichopodidae (Diptera)
}

\author{
MARIA ALEXANDROVNA CHURSINA \\ Voronezh State University, Universitetskaya sq., 1, 394006 Voronezh, Russia. \\ email: chursina.1988@list.ru \\ Manuscript received: 25 June 2019. Revision accepted: 13 August 2019.
}

\begin{abstract}
Chursina MA. 2019. Convergent evolution of sexual dimorphism in species of the family Dolichopodidae (Diptera). Biodiversitas 20: 2480-2485. In this study, we investigated patterns of sexual dimorphism in four dolichopodid species namely Dolichopus acuticornis Wiedemann, 1817, D. longicornis Stannius, 1831, Rhaphium appendiculatum Zetterstedt, 1849, and R. caliginosum Meigen, 1824 that are exhibiting similar patterns of sexual dimorphism. Although they are distantly related, males of all these species exhibit similar sexual dimorphic characters in term of elongation of postpedicel. A comparison of variation made it possible to assumed that although sexual selection acts differently, all four species exhibited similar patterns of sexual dimorphism in wings and legs; females possessed relatively widened wing and elongated fore and hind femora than males. Cluster analysis for the morphometry data demonstrated that the differences between sexes significantly exceeds the differences between species. These results may reflect the presence of some conservative genetic structure of covariation among characters.
\end{abstract}

Keywords: Antennal morphology, Dolichopodidae, legs morphology, sexual dimorphism, wing shape

\section{INTRODUCTION}

Sexual dimorphism in antennal size and shape often occurs in dipteral insects. In most cases, the essential differences are caused by the fact that one sex, for instance, males, is adapted to perceptive pheromones or acoustic signals (Sivinski 1997). Therefore, the great diversity of antennal shape divergence in dolichopodid flies is widely used as a species-diagnostic character (Negrobov et al 2015). Sexual dimorphism in antennal was also evident, and increasing the length of postpedicel in males is particularly widespread to males from the family. Postpedicel of males of some genera is much longer than its width at the base while female postpedicel as long as wide.

It is noteworthy that non-related dolichopodid species exhibited a similar pattern of sexual dimorphism in antennal morphology. For instance, elongation of males postpedicel is observed in species in the genus Dolichopus Latreille, 1796, Rhaphium Meigen, 1803, Syntormon Loew, 1857, Systenus Loew, 1857 and others (Negrobov et al. 2015). The presence of a similar pattern of sexual dimorphism in distantly related species helps us appreciate whether there are any conserved genetic factors of sexual dimorphism, which leads to stronger convergence than it was to be expected in response to selective pressure alone.

For testing this hypothesis, we estimated the intraspecific sexual variation on the antennal, wings, and legs morphometry and compared sex-related variation of four species of the Dolichopodinae and Rhaphiinae subfamilies, belonging to the genera Dolichopus ( $D$. acuticornis Wiedemann, 1817 and D. longicornis Stannius, 1831)and Rhaphium (R. appendiculatum Zetterstedt, 1849 and $R$. caliginosum Meigen, 1824). These species have similar size and ecological requirements: they prefer moist and shadow habitats, are common on the banks of forest rivers, in riparian vegetation and wetlands. Both $D$. acuticornis and D. longicornis also both $R$. appendiculatum and $R$. caliginosum are supported as closely related species in the basis of study with using DNA data (Bernasconi et al. 2007a, b). As described by Stackelberg (1933), postpedicel of Dolichopus acuticornis males almost two times as long as width, one of $D$. longicornis more than two times as long as width, and Rhaphium species are characterized by the longest postpedicel among the species represented, it is almost 5 times as long as width.

\section{MATERIALS AND METHODS}

In general, 303 specimens of the following species were used: Dolichopus acuticornis (13 males and 31 females), D. longicornis (68 males and 36 females), of Rhaphium appendiculatum (18 males and 29 males), $R$. caliginosum ( 33 males and 75 females). Specimens were collected by hand net in June - August 2018, and deposited in the entomology collection of Voronezh State University (VSU). Each species was represented by specimens from the one natural population: $D$. acuticornis and $D$. longicornis were sampled in village Ramon, Voronezh region $\left(51^{\circ} 54^{\prime} 25^{\prime \prime} \mathrm{N} 39^{\circ} 21^{\prime} 12^{\prime \prime} \mathrm{E}\right), R$. appendiculatum in Botanic Garden of VSU (51 $\left.42^{\prime} 47^{\prime \prime N} 39^{\circ} 12^{\prime} 16^{\prime \prime E}\right), R$. caliginosum in village Mitrofanovka, Voronezh region (495' $\left.15^{\prime \prime} \mathrm{N} 39^{\circ} 41^{\prime} 0 " \mathrm{E}\right)$.

The wings, legs, and antennae were separated from the body, placed on a microscope slide under a coverslip and 
photographed with Levenhuk C310 NG microscope digital camera. To eliminate digitizing error, all wings were digitized two times. It was shown that effect of measurement error was less than $1 \%$ of the total variation and can be neglected. Measurement repeatability was very high.

Wing shape was studied using the landmark-based geometric morphometric method (Klingenberg and McIntyre 1998; Zelditch et al. 2004). Nine homologous landmarks (Bookstein 1991) positioned at vein junctions and terminations (Figure 1). Digitalization of the landmarks was made using the software tpsUtil and tpsDig (Rohlf 2013a, b). Then a generalized Procrustes analysis (GPA) and morphometric analysis were performed using MorpholJ software (Klingenberg 2011). The resulting set of variables (Procrustes coordinates) were used for future analysis.

Nine morphometric characters of the legs and three morphometric characters of antennae were measured (Figure 1): lengths of wing fore, middle, and hind femora $\left(F_{1}, F_{2}, F_{3}\right)$, fore, middle, and hind tibia $\left(T_{1}, T_{2}, T_{3}\right)$, the first segment of fore, middle and hind tarsi $\left(\operatorname{tar}_{1}, \operatorname{tar}_{2}, \operatorname{tar}_{3}\right)$; length and height of postpedicel $\left(\mathrm{L}_{\mathrm{pp}}\right.$ and $\left.\mathrm{H}_{\mathrm{pp}}\right)$, and length of arista-like stylus $\left(\mathrm{L}_{\mathrm{ar}}\right)$.

Test for significant differences among species and sexes were undertaken using a multivariate analysis of variance (MANOVA). Canonical variate analysis (CVA) was applied to describe the differences between groups, the hierarchy of which was then determined using the unweighted pair group method with arithmetic mean (UPGMA) using Mahalanobis interspecific distances. All statistical analyses made with the Statistica (StatSoft Inc. 2012) and Past software (Hammer 2001).

We evaluated the overall similarity of patterns of sexual shape dimorphism of the studied species by Mantel test (Mantel 1967); the statistical significance of the results was assessed by the permutation test with 1000 replicates. In addition, we performed principal component analysis (PCA) both on the matrix of Procrustes coordinates and on the matrix of all morphometric characters to reduce the dimensionality of the data and to the identification of main characters of variation.

Because the first component of wing shape variation associated with sexual dimorphism accounted for most of the total shape variation (more than $70 \%$ ), we compared them to describe the similarity in sexual shape dimorphism. For this purpose, we tested the null hypothesis stating that the PC vectors for different species were identical. This null hypothesis was tested by bootstrap analysis with 10 000 replicates.

Than PCA was performed on the general matrix of morphometric characters for both combined matrix (males and females) and for sexes separately. For each pair of first principal component, we computed Spearman rank correlation (rs) to compare, first, the pattern of total phenotypic variation in males and females of one species, secondly, in males and females of different species, and, thirdly, to compare the patterns of sexual dimorphism of each pair species. Because the distribution of some characters was different from normal, we used nonparametric tests.

\section{RESULTS AND DISCUSSION}

The differences in legs and wing morphometry both between sexes and among species were statistically significant (Table 1). The significant species and sex interaction show the difference in the patterns of sexual dimorphism between species. Significant differences remained when analyzing the matrix of wing and legs characters separately.
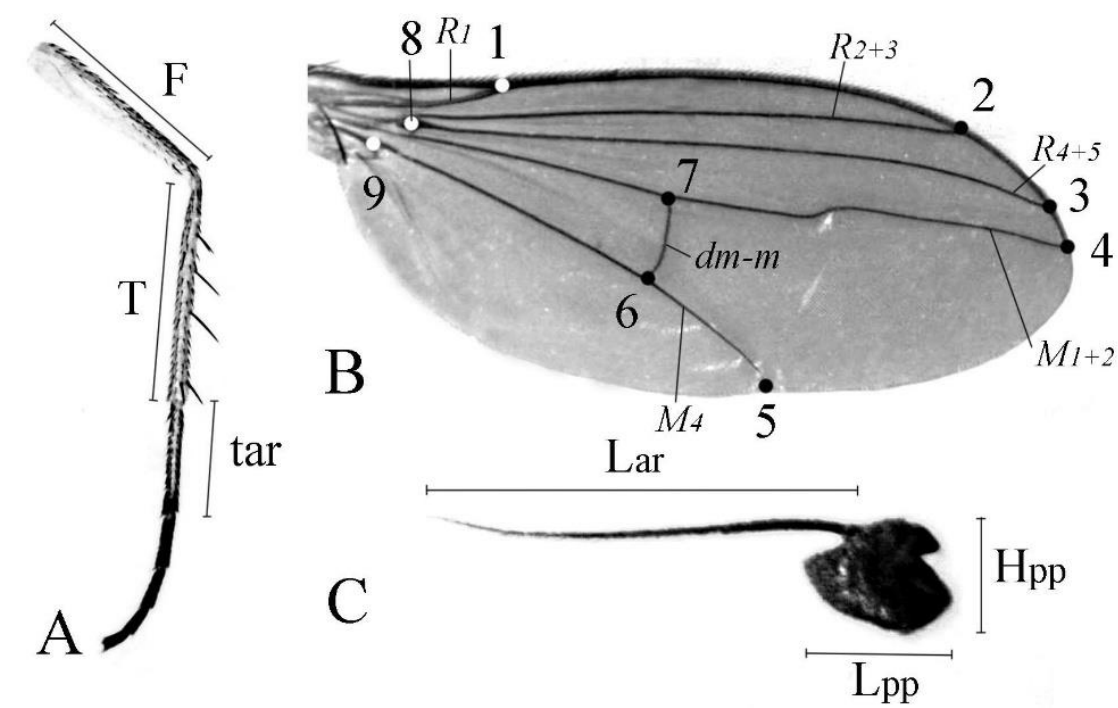

Figure 1. Characters used in the study (Dolichopus acuticornis, female): A. fore leg; B. wing with nine landmarks; C. postpedicel and arista-like stylus. The nomenclature carried out as per Grichanov and Brooks (2017) 


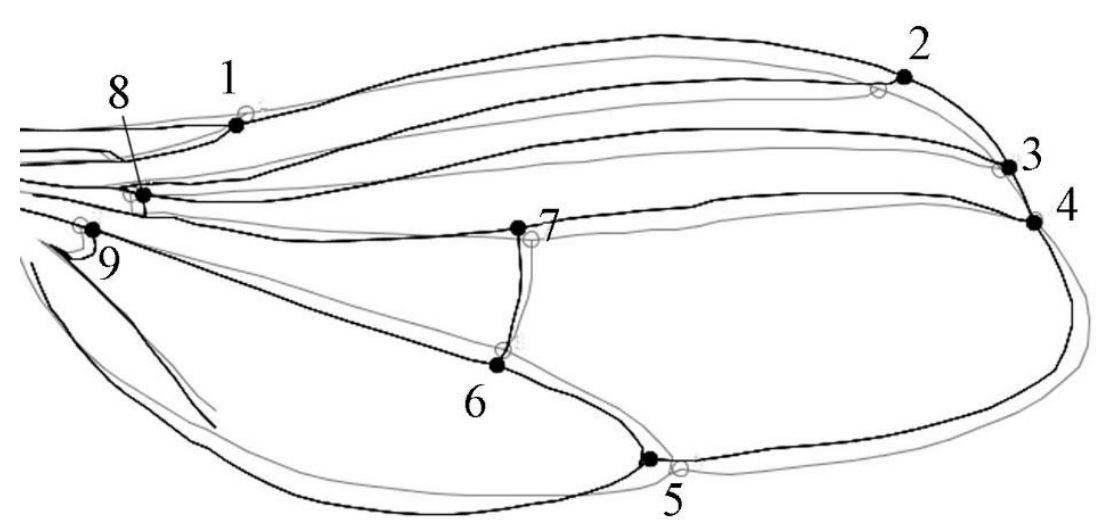

Figure 2. Wing shape changes associated with sexual dimorphism (Dolichopus acuticornis example): grey outline: the average shape of male, black outline: the average shape of female

Table 1. The effect of species, sex, and their interaction on wing and legs morphometric characters for four Dolichopodidae species (results of MANOVA).

\begin{tabular}{lccccc}
\hline \multicolumn{1}{c}{ Effect } & $\begin{array}{c}\text { Wilk's } \\
\text { Lambda }\end{array}$ & F & $\begin{array}{c}\text { Df } \\
\text { num }\end{array}$ & Df den & P \\
\hline Species & 0.0006 & 80 & 63 & 466.5 & $<0.0001$ \\
Sex & 0.1621 & 38 & 21 & 156.0 & $<0.0001$ \\
Species $^{\times}$sex & 0.0686 & 11 & 63 & 466.5 & $<0.0001$ \\
\hline
\end{tabular}

Note: Df num: degrees of freedom for the numenator, Df den: degrees of freedom for the denomenator, P: P-level.

CVA of Procrustes coordinates indicated that sexual dimorphism for all studied species is affected mostly landmarks, defining anterior wing margin (landmark 2), and posterior crossvein with apical part of $M_{4}$ (landmarks 5, 6 and 7) (Figure 2). Females possessed by far longer posterior crossvein and $R_{2+3}$ and shortened apical part of $M_{4}$.

All four covariance matrices of sexual dimorphism have statistically significant correlations (Table 2). The most similarity of the pattern of wing sexual dimorphism was observed for the pairs Dolichopus acuticornis and D. longicornis, Rhaphium caliginosum and Dolichopus acuticornis, Rhaphium caliginosum and D. longicornis. Additionally, both correlation analysis and calculating the angles between the first PCs of sexual dimorphism showed that the biggest difference exists between patterns of sexual dimorphism of Rhaphium appendiculatum and Dolichopus acuticornis.

Cluster analysis for the wing morphometry data revealed that although the magnitude of difference between sexes is significantly lower in Rhaphium species than in Dolichopus species, the differences between males and females in both cases exceed the interspecific differences. In other words, the wing shape of males of two different species is more similar than the wing shape of females and males of the same species (Figure 3).

Sexual dimorphism in legs morphometry of all studied species is that females have longer fore and hind femora than males. Cluster analysis for the legs morphometry data also demonstrated that the differences between sexes significantly exceed the differences between species and even the differences between genera (Figure 4). According to this analysis, two groups can be identified: the first group included females of all species, and the second group included males. The greatest similarity in legs morphometry has been found between the following pairs: females of Dolichopus longicornis and Rhaphium caliginosum, males of Dolichopus acuticornis and D. longicornis.

The species showed a similar magnitude of difference between sexes. The degree of sexual dimorphism (the ratio of the mean female trait to mean male trait) for the length of $d m-m$ ranged from 1.07 (D. acuticotnis) to 1.11 ( $R$. caliginosum); of fore femora length ranged from 1.04 ( $D$. acuticotnis) to 1.10 (R. appendiculatum).

The first PCs computed on the matrix of morphometric characters including the wing, legs, and antennal morphometric characters were compared to examine the general similarity of variation. Spearman rank correlation conducted on the first PCs of sexual dimorphism demonstrated their significant similarity for both species Rhaphium and Dolichopus (Table 3, under the main diagonal), whereas there was no significant similarity between species of different genera.

Within each species, highly significant similarity between intrasexual variability of males and females are obtained (Table 3, the main diagonal). Statistically significant correlations were found between intragroup variations of females of all studied species, while the largest similarity was exhibited by $R$. appendiculatum and $D$. longicornis females. There was no correlation between variation of both $R$. appendiculatum and $D$. acuticornis males and $R$. caliginosum and $R$. appendiculatum males.

We observed a high negative correlation in $D$. acuticornis male between postpedicel length and length of all studied legs segments (for instance, rs $\left(L_{p p}\right.$ and $\left.T_{l}\right)=-$ $0.69)$, that is, males with relatively longer postpedicel tend to have relatively shorter legs; whereas a significant relationship between postpedicel length and wing characters was not found. Postpedicel length was correlated with the length of the first segment of fore tarsi in $R$. appendiculatum males ( $\mathrm{rs}\left(L_{p p}\right.$ and $\left.\left.\operatorname{tar}_{1}\right)=0.74\right)$. 


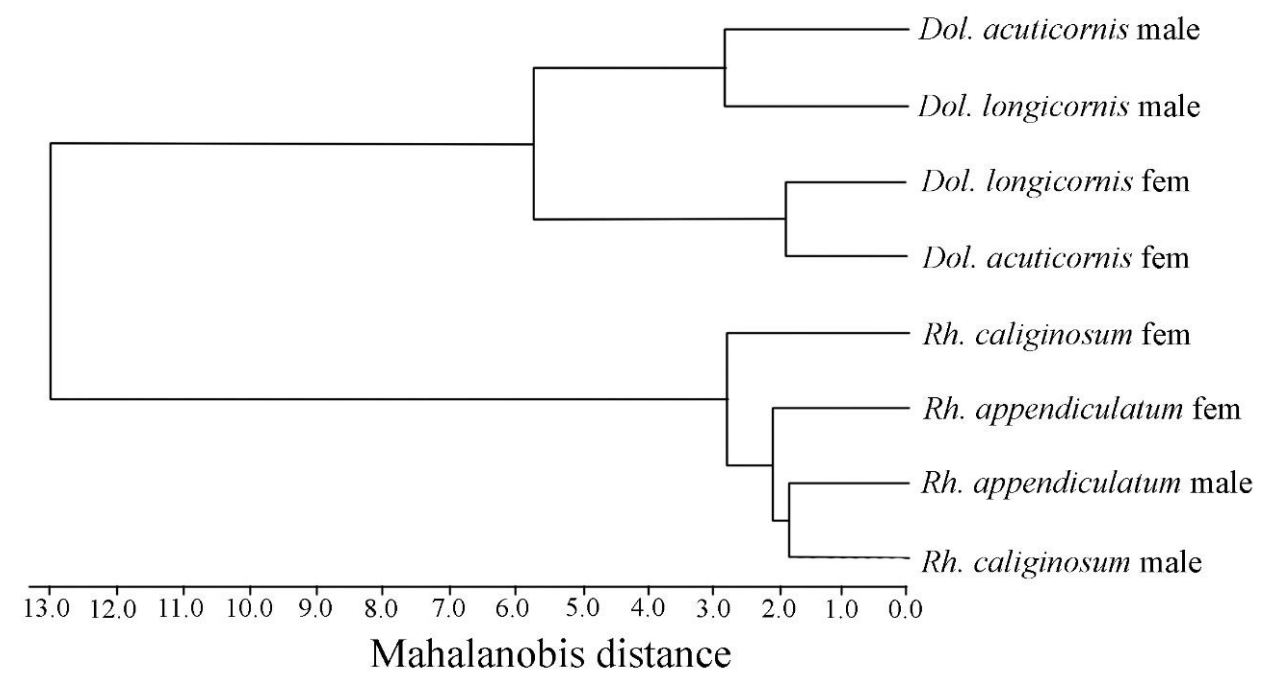

Figure 3. UPGMA dendrogram showed similarity of wing shape among males and females of four Dolichopodidae species

Table 2. The matrix correlations between covariance matrices of sexual dimorphism (over the main diagonal) and the angles between the first PCs of sexual dimorphism (under the main diagonal) for four Dolichopodidae species

\begin{tabular}{lllll}
\hline & $\mathbf{1}$ & $\mathbf{2}$ & $\mathbf{3}$ & $\mathbf{4}$ \\
\hline D. acuticornis & & $0.94^{*}$ & $0.68^{*}$ & $0.91^{*}$ \\
D. longicornis & $10.67^{\circ *}$ & & $0.79^{*}$ & $0.91^{*}$ \\
R. appendiculatum & $59.85^{\circ}$ & $35.81^{\circ *}$ & & $0.60^{*}$ \\
R. caliginosum & $20.28^{\circ *}$ & $14.93^{\circ *}$ & $66.46^{\circ}$ & \\
\hline
\end{tabular}

Note: *: $\mathrm{P}<0.0001$

The opposite trend occurred in D. longicornis males; there was a moderate correlation between postpedicel length and single legs' segments: rs $\left(L_{p p}\right.$ and $\left.\operatorname{tar}_{2}\right)=0.50$, rs $\left(L_{p p}\right.$ and $\left.F_{3}\right)=0.44 ;$ rs $\left(L_{p p}\right.$ and $\left.\left.T_{3}\right)=0.43\right)$. Postpedicel length is moderate positively correlated with wing characters, such as length of the longitudinal vein in $R$. caliginosum: $\mathrm{rs}\left(L_{p p}\right.$ and $\left.\mathrm{R}_{4+5}\right)=0.57$; $\mathrm{rs}\left(L_{p p}\right.$ and $\left.\mathrm{M}_{4}\right)=$ $0.50)$.

\section{Discussion}

Sexual dimorphism is a widespread source of phenotypic variation in the Dolichopodidae family; in particular, dolichopodid males often bear sexually dimorphic antennae. For example, males of Sybistroma Meigen, 1824 have one or several enlargements on the arista-like stylus; Dolichopus, Hercostomus Loew, 1857 and Poecilobothrus Mik, 1878 males sometimes have elongated postpedicel and weakly or strongly pubescent arista-like stylus. There are also less obvious sexual dimorphic characters in the family Dolichopodidae, for example, sexual dimorphism in wing shape (Chursina and Negrobov 2018) and legs morphometry.

This study is aimed primarily at the comparison of patterns of sexual dimorphism in four distantly related dolichopodid species, exhibiting sexually dimorphic postpedicel elongation in males. If the similarity of males variation of different species would be higher than the similarity of variation within species, it would be possible to assume that there are some patterns of variation determined either by functional requirements (for example, when the postpedicel increases, the wing shape changes) or epigenetic structure (Vasiliev et al. 2018).

Comparative analyses were conducted on wing-vein landmarks and legs morphometric characters. It is clear from the results that male and female differ not only in the size of the postpedicel, but also in the wing shape and length of femora. A common character of wing shape dimorphism is an elongation of $R_{2+3}$, and $d m-m$, and shortened apical part of $M_{4}$ from male to female. We found also a certain similarity in sexual dimorphism of legs morphometry: relative elongation of fore and hind femora in females than in males. Both in wing shape and legs morphometric characters, males of different species showed greater similarity than males with females of the same species (Figures 3-4).

The species demonstrated not only congruent patterns of variation associated with sexual dimorphism but also similar degrees of dimorphism. We believe that sexual selection pressure acts so differently on Dolichopus and Rhaphium males that cannot explain the observed degree of phenotypic convergence. It is recognized that Dolichopus males use wings and legs in performing the mating dance to attract females (Stubbs 1988; Land 1993). The male faces the female and vibrates its wings and demonstrated its legs. On one occasion, male and female were observed contacting their antennae. In Rhaphium species, there are no obvious elements of such mating. The male dives on a resting female from above, and if the female is not ready to mate, she drives it away using her wings. Therefore, in Rhaphium species, male body shape is not under strong sexual selection through female mate choice. Relatively longer wings in Rhaphium males can be explained by the need to be more maneuverable. 


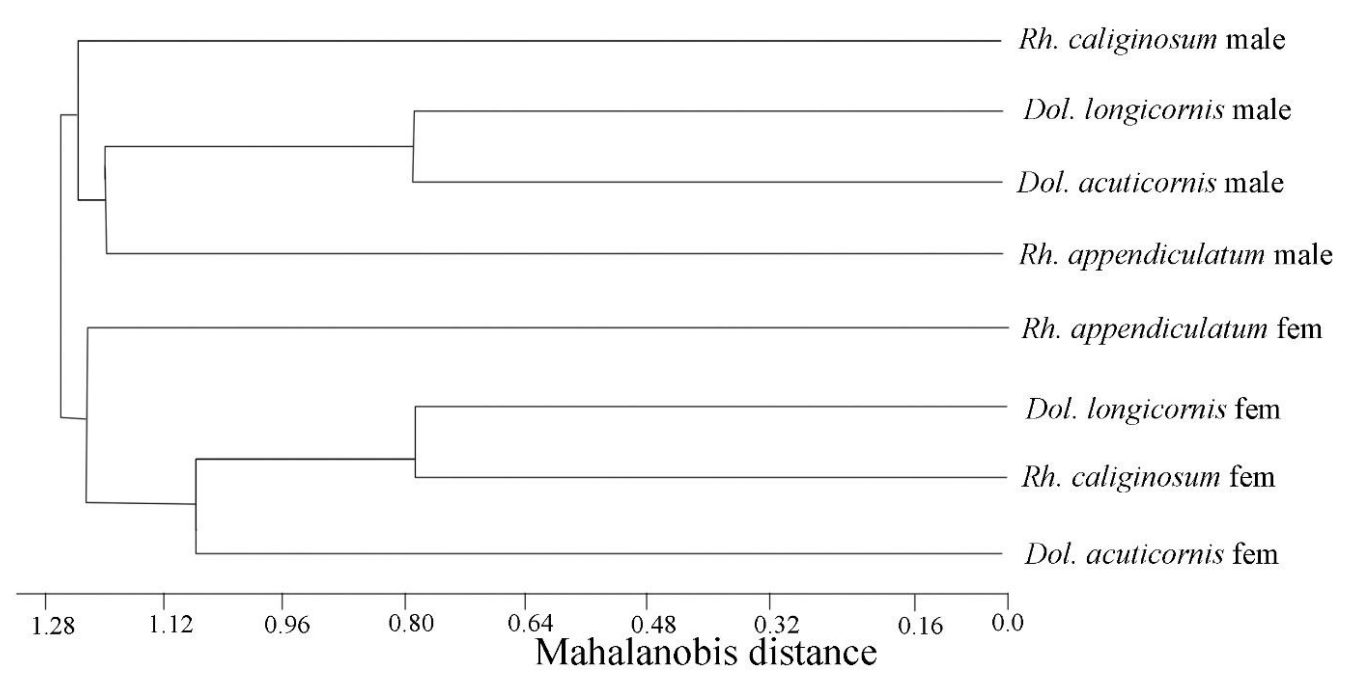

Figure 4. UPGMA dendrogram showed similarity of legs morphometry among males and females of four Dolichopodidae species

Table 3. Spearman rank correlation between first PCs of sexual dimorphism of wing, legs and antennae morphometric characters (under the main diagonal); Spearman rank correlation between first PCs of intrasexual variability of males and females (the main diagonal); Spearman rank correlation between first PCs of males/females variability (over the main diagonal) for four Dolichopodidae species

\begin{tabular}{lllll}
\hline Species & $\mathbf{1}$ & $\mathbf{2}$ & $\mathbf{3}$ & $\mathbf{4}$ \\
\hline D. acuticornis & $0.64^{*}$ & $0.85^{*}$ & 0.43 & 0.52 \\
D. longicornis & $0.84^{*} / 0.59^{*}$ & $0.87^{*}$ & 0.39 & 0.47 \\
$R$. appendiculatum & $0.32 / 0.60^{*}$ & $0.66^{*} / 0.89^{*}$ & $0.66^{*}$ & $0.87^{*}$ \\
$R$. caliginosum & $0.87^{*} / 0.70^{*}$ & $0.88^{*} / 0.84^{*}$ & $0.49 / 0.80^{*}$ & $0.83^{*}$ \\
\hline Note: $*$ : $<<0.001$ & \multicolumn{6}{l}{}
\end{tabular}

At this stage of the investigation, it is more difficult to explain the elongation of femora in females based on functional requirements. From the other studies, it is known that legs elongation occurs as a sexually dimorphic character in other dipteral (Bonduriansky 2006). However, in this case, legs elongation is linked with antennal and head elongation, which Telostylinus angusticollis and Prochyliza xanthostoma males use in male-male combat. Whereas in Dolichopus and Rhaphium flies, the leg elongation correlated with decreasing of postpedicel (in females), and legs do not participate directly in males sexual behavior.

Thus, the sexual behavior of Dolichopus and Rhaphium species has no similar features, due to which identify patterns of sexual dimorphism might evolve. Sexual selection does not quantify that the antennae are the most dimorphic organs in the species from the different subfamilies, wings are at the second place, and fore and hind femora are in third place. The least dimorphic characters were the other legs' segments.
Then we carried out a correlation analysis within the species to assess how the studied characters are related. Table 3 shows that females more often exhibited similar patterns of the variance than males, whereas the variance of males was distributed in different dimensions, and this fact can be related to stronger selection pressure exerted on males.

Taking into account the ecological similarity of studied species and their similar body sizes $(3.5-4 \mathrm{~mm})$, this can be assumed that interspecific similarity reflects similar functional requirements. For instance, it has been suggested, that elongation of postpedicel can be related to a change in wing shape to compensate for flight ability. However, changes to the postpedicel length were linked to changes in the wing shape only in Rhaphium caliginosum, in other cases together with the changes in the antennal morphology, changes in legs morphometric characters occurred. On the other hand, since the distantly related species showed similar patterns of sexual dimorphism in wing shape and legs morphometry, one may reflect the presence of some conservative genetic covariance structure (Emlen and Nijhout 2000).

In conclusion, similar patterns of sexual dimorphism in non-closely related taxa are poorly studied, and they were considered, mainly, for stalked-eyed dipterans (Wilkinson and Dodson 1996). Further researches in this field can be extended to species exhibiting one type of sexual dimorphism (for example, extended segments of the tarsi, modified middle tibia, etc.). The results obtained in this direction will help assess the presence of conservative genetic structures or phenomena of pleiotropy, that is, the formation of a complex of associated traits with the response to selection acting on only one line. 


\section{ACKNOWLEDGEMENTS}

The author would like to thank two anonymous reviewers for their constructive comments. I thanks also to Prof. O.P. Negrobov, Voronezh State University, Russia kindly helped confirm the identification of the studied species.

\section{REFERENCES}

Bernasconi MV, Pollet M, Varini-Ooijen M, Ward PI. 2007a. Molecular systematics of Dolichopodidae (Diptera) inferred from COI and 12S rDNA gene sequences based on European exemplars. Invertebr Syst 21: 453-470. DOI: 10.1071/IS06043

Bernasconi MV, Pollet M, Varini-Ooijen M, Ward PI. 2007b. Phylogeny of European Dolichopus and Gymnopternus (Diptera: Dolichopodidae) and the significance of morphological character inferred from molecular data. Eur J Entomol 104: 601-607. DOI: 10.14411/eje.2007.075

Bookstein FL. 1991. Morphometric Tools for Landmark Data: Geometry and Biology. University Press, Cambridge.

Bonduriansky R. 2006. Convergent evolution of sexual dimorphism in Diptera. J Morphol 267: 602-611. DOI: 10.1111/evo.12100

Chursina MA, Negrobov OP. 2018. Phylogenetic signal of the wing shape in the subfamily Dolichopodinae (Diptera, Dolichopodidae). Ent Rev 98: 515-527. DOI: 10.7868/S0044513418060041

Emlen DJ, Nijhout HF. 2000. The development and evolution of exaggerated morphologies in insects. Annu Rev Entomol 45: 661708

Grichanov IY, Brooks SE. 2017. Dolichopodidae (Long-legged flies). In Kirk-Spriggs AH, Sinclair BJ (eds). Manual of Afrotropical Diptera. Volume 2. Nematocerous Diptera and lower Brachycera. Suricata 5. South African National Biodiversity Institute, Pretoria.
Hammer O. 2001. PAST: Paleontological Statistics Software Package for Education and Data Analysis. Palaeontol Electron 4:1-9.

Klingenberg CP. 2011. MorphoJ: an integrated software package for geometric morphometrics. Mol Ecol Resour 11: 353-357. DOI: 10.1111/j.1755-0998.2010.02924.x.

Klingenberg CP, McIntyre GS. 1998. Geometric morphometrics of developmental instability: analyzing patterns of fluctuating asymmetry with Procrustes methods. Evolution 52: 1363-1375. DOI: $10.2307 / 2411306$

Land MF. 1993. Chasing and pursuit in the dolichopodid fly Poecilibothrus nobilitatus. J Comp Physiol 173: 605-613.

Mantel N. 1967. The detection of disease clustering and a generalized regression approach. Cancer Res 27: 209-220.

Negrobov OP, Chursina MA, Selivanova OV. 2015. Antennal morphology in the family Dolichopodidae (Diptera). J Insect Biodivers 3: 1-10. DOI: $10.12976 / \mathrm{jib} / 2015.3 .8$

Rohlf FJ. 2013a. TpsUtil, file utility program. version 1.60. Department of Ecology and Evolution, State University of New York at Stony Brook.

Rohlf FJ. 2013b. TpsDig2, digitize landmarks and outlines, version 2.17. Department of Ecology and Evolution, State University of New York at Stony Brook.

Sivinski J. 1997. Ornaments in the Diptera. Fla Entomol 80: 142-164.

Stackelberg AA. 1933. 29. Dolichopodidae. In: Lindner E (eds). Die Fliegen der Palaearktischen Region. Schweizerbart, Stuttgart.

StatSoft Inc. 2002. STATISTICA-data analysis software system, Version 6. [Computer software]. StatSoft, USA.

Stubbs A. 1988. Courtships of Dolichopus plumipes (Scop) Dolichopodidae). Dipt Dig 1: 43.

Vasiliev AG, Vasilyeva IA, Shkurikhin AO. 2018. Geometric morphometry. From theory to practice. KMK, Moscow. [in Russian]

Wilkinson GS, Dodson GN. 1996. Function and Evolution of Antlers and Eye Stalks in Flies. In: Choe J, Crespi B (eds). The evolution of mating systems in insects and arachnids. Cambridge University Press, Cambridge.

Zelditch ML, Swiderski DL, Sheets HD. 2004. Geometric Morphometrics for Biologists: A Primer. Academy Press-Elsevier, New York. 